\title{
Décentralisation et régionalisme face au système politique centralisé en République Démocratique du Congo. Problèmes et attentes
}

\author{
Par NKWANDA MUZINGA Simplice*
}

\section{INTRODUCTION}

A la lecture de la constitution, spécialement à ses articles 2, 3, 201, 202, 203 et 204, il s'avère que le constituant a restructuré administrativement l'Etat en créant des nouvelles provinces qu'il s'est résolument engagé dans la voie d'un partage des compétences entre le pouvoir central et les provinces. Ainsi, il a accordé aux provinces et aux entités territoriales décentralisées (E.T.D.), la personnalité juridique, la libre administration et l'autonomie de gestion.

La tournure employée par le constituant aux alinéas 1 et 2 de l'article 3 évidemment que la province n'est pas une entité territoriale décentralisée au sens de la décentralisation strictement entendue. L'article 2 de la loi 08/012 du 31 juillet 2008 portant principes fondamentaux relatifs à la libre administration des provinces, considère la province comme une composante politique et administrative du territoire de la République. Elle a donc en vertu de la constitution une autonomie plus large dépassant celle dévolue aux entités territoriales décentralisées, mais ses rapports avec le pouvoir central restent discutables. Ce qui constitue un achoppement à l'application et à la réussite du processus de la décentralisation prônée par le constituant.

Toutefois, l'exposé des motifs de la loi numéro 08/012 du 31 juillet 2008 ci-haut citée, renseigne sur le statut, l'organisation et le fonctionnement de la province possédant des dispositions constitutionnelles qui instituent le régionalisme politique en République Démocratique du Congo. Pourtant la constitution évoquée n'en parle pas nettement mais au moins détermine les institutions politiques de la province et répartie les compétences entre le pouvoir central et les provinces.

Politiquement, il faut décentraliser pour développer l'organisation des collectivités territoriales en favorisant l'émergence d'un Etat de droit démocratique et la promotion des droits de l'homme.

Administrativement, il faut décentraliser pour développer l'organisation et la gestion des ressources naturelles, financières et techniques de nos collectivités territoriales.

Socialement et culturellement, il faut décentraliser pour développer ce pays. En effet, les pouvoirs publics au niveau national, provincial et local doivent associer la société civile dans la gestion et le contrat de la République Démocratique du Congo. La responsabilité de la société civile porterait non seulement sur les actions de participation effective, mais aussi

\footnotetext{
* Docteur en Droit Canon et Professeur Associé à l'UNILU.
} 
et surtout sur elle-même, de contrôle efficace dont elle dispose dans une société politiquement organisée.

S'agissant de la participation des groupes culturellement et socialement marginalisés dans la prise de décision, il faut savoir que : « la participation sans exclusion de tous les citoyens à l'œuvre du développement collectif, est un principe démocratique au centre de la démocratie locale.

Quelle économie de la compréhension de l'applicabilité de la décentralisation et du régionalisme constitutionnel par rapport au système politique centralisé et ses problèmes, quelles sont les pistes de solutions dans l'avenir?

- Quelles sont les attentes de la population au regard de la décentralisation, de la régionalisation et du découpage territorial face au système politique centralisé?

- Que faire?

\section{A. ETAT DE LIEUX ET POSITION DU PROBLEME}

Le régionalisme constitutionnel dans la constitution du 18 février 2006 pose deux problèmes majeurs : un fédéralisme masqué d'une part et d'autre part l'existence de la centralisation qui confond encore qui couvre et met en jeux des principes de la décentralisation territoriale technique et fonctionnelle au profit d'une autorité agissant politiquement et coordonnant tous les pouvoirs dans ses mains.

Pour SIMON METENA M'NTEBA, la décentralisation n'est pas comme d'aucuns seraient tentés de le croire ou veulent nous faire accroître, une « mince affaire » ou du " gâteau ». C'est un terrible mécanisme où des intérêts titanesques et non moins les titans qui les représentent, les défendent ou les pourfendent devront s'affronter, serein enfin de le faire triompher ou choisir selon un marchandage régulé ${ }^{1}(1)$.

En effet, pour développer la République Démocratique du Congo, ma conviction profonde est qu'il faut la décentraliser politiquement, administrativement, socialement et culturellement. Politiquement, il faut décentraliser pour développer l'organisation des collectivités territoriales en favorisant l'émergence d'un Etat de droit démocratique et la promotion des droits de l'homme ${ }^{2}(2)$.

La plupart des décentralisations qui ont eu lieu dans la décennie passée, ont été motivées par des raisons purement politiques. En Amérique latine par exemple, la décentralisation a une partie essentielle du processus de la démocratisation.

En Afrique, la propagation du pluralisme politique a créé une demande pour une plus grande participation locale dans les prises des décisions.

1 (1)Simon-Pierre METENA, Des « Entités territoriales décentralisées (E.T.D.). Qu'est-ce à dire et pourquoi faire? », In Congo Afrique n` 433 mars 2009, Lubumbashi, p.p. 187 à 202.

2 (2)Félix VUNDUAWE té Pemako, la dynamique de la décentralisation territoriale en République Démocratique du Congo, Afrique $n^{\circ}$ 433, Mars 2009, Lubumbashi, p.p. 165 à 185. 
Dans certains pays comme l'Ethiopie, la décentralisation a eu lieu en réponse aux pressions exercées par des groupes régionaux ou ethniques pour avoir davantage le contrôle ou de participation dans le processus politique.

A l'extrême, la décentralisation représente une tentative d'espèce pour préserver l'unité du pays face à ce genre des pressions en accordant plus l'autonomie dans toutes les régions ou en forgeant « des formations asymétriques ».

Dans d'autres contextes, la décentralisation a simplement eu lieu en l'absence d'autre structure d'administration efficace pour fournir les services publics locaux. En Asie de l'Est, la décentralisation paraît remotivée par le besoin d'améliorer la fourniture des services, des grandes populations et par la reconnaissance des limites de ce que peut faire l'administration centrale.

Si la décentralisation ne peut être confondue avec le fédéralisme, elle n'est pas non plus synonyme du régionalisme politique ou constitutionnel. Celui-ci constitue également un procédé d'aménagement du pouvoir d'Etat qui constitue à décentraliser politiquement et constitutionnellement les provinces, qui dans un Etat unitaire des composantes politiques et administratives dotées de la personnalité juridique et l'autonomie institutionnelle et financière. $^{3}(3)$.

Par rapport à la décentralisation, l'originalité du régionalisme réside dans le fait que la répartition des compétences entre le pouvoir central et les provinces ainsi que l'ordre institutionnel de celles-ci sont définis par la constitution et non par une loi organique qui peut à tout moment être modifié par la seule volonté du législateur. Pour éviter les interventions intempestives du centre sur la périphérie, la loi fondamentale prend le soin de distinguer clairement, un peu comme dans le fédéralisme.

En définitive, le régionalisme constitutionnel traduit clairement ce que d'aucun appellent « un Etat unitaire fortement décentralisé ». C'est donc une formule de gestion intermédiaire entre un Etat unitaire décentralisé et un Etat fédéral.

Mais, les trois systèmes de gouvernement (décentralisation, fédéralisme et le régionalisme politique) reposent tous sur le principe de la libre administration.

\section{B. DECENTRALISATION ET REGIONALISME EN RDC}

\section{LA DECENTRALISATION}

La décentralisation est le système d'origine administrative dans lequel il y a création par la loi ou en vertu de la loi, en dehors du centre d'autres niveaux de responsabilité et de décision. Elle consiste donc à confier des pouvoirs de décisions à des organes autres que de

3 (3)J. MEKHANTAR, Droit politique et constitutionnel, Paris, 1997, p. 51. Lire aussi X. PHILIPPE, «La répartition des compétences entre l'Etat central, l'Etat provincial et les municipalités : Structures politiques ou administrative? » In Revue française de l'administration publique, $n^{\circ} 85$, Janvier-Mars 1998, p.p. 15-35. 
simple agent du pouvoir central, élus démocratiquement par les citoyens de la collectivité intéressée.

La décentralisation repose sur la reconnaissance par le pouvoir central d'intérêt distinct et de ceux du gouvernement de l'Etat. Quel que soit la forme de la décentralisation celle administrative se caractérise par l'existence d'un pouvoir de contrôle administratif des autorités, ce qui signifie que les collectivités décentralisées n'ont aucun pouvoir de s'organiser elles-mêmes ${ }^{4}(1)$.

Il faut noter que la décentralisation est un facteur de bon fonctionnement de l'administration puisque celle-ci pourra, s'agissant d'affaire locale ou spéciale, prendre des décisions rapides adaptées aux circonstances et en pleine connaissance des besoins des administrés dont elle se trouve rapproché.

Le constituant du 18 février 2006 opté pour la décentralisation comme mode de gestion de certaines entités territoriales (E.T.) de la République que la loi organique $\mathrm{n}^{\circ}$ 8-016 du 07 octobre 2008 n'est en fait que la concrétisation aboutie de l'énonce déjà faite du constituant à l'article 3, alinéa 2 et 4 de la constitution d'élaborer une loi organique devant fixer les règles relatives à la composition, à l'organisation et au fonctionnement des entités territoriales décentralisées (E.T.D.) et de leurs rapports avec l'Etat et les provinces ${ }^{5}(2)$. Dans son discours qu'il prononça à l'Université Nationale de Ruanda, Maître Kapya Kabesa a stigmatisé que la décentralisation n'est pas liée aux apparences mais au développement économique des Etats selon les lieux, les régions, le temps et l'histoire ${ }^{6}(1)$.

\section{Type de décentralisation}

On distingue la décentralisation territoriale et la décentralisation technique.

Pour simplifier la discussion, nous retiendrons la définition selon laquelle la décentralisation est un mode de gestion des services publics qui se traduit par le transfert d'attribution de l'Etat à des institutions juridiquement distinctes de lui et bénéficiant en vertu d'une loi organique d'une certaine autonomie ${ }^{7}(2)$.

Tout en n'ayant pas la prétention d'être la plus complète, cette définition présente l'avantage de contenir en elle les principaux éléments constitutifs de la décentralisation : la personnalité juridique, l'existence des matières locales et l'autonomie reconnue par une loi organique. Elle permet en outre de distinguer les deux types classiques de la décentralisation, à savoir :

4 (1) BAGAHUA MUHEME, Economie régionale par la gestion décentralisée des projets. Academia - Bruylant, Louvain-la-Neuve 2005, p. 30.

5 (2) Simon-Pierre METENA M'NTEBA, « Des entités territoriales décentralisées (E.T.D.) qu'est-ce à dire et pourquoi faire? », In Congo-Afrique $n^{\circ}$ 433, Mars 2009, L'shi, p.p. 187-202.

6 (1) Kapya Kabesa, " la décentralisation au Ruanda, au Burundi et en R.D.C., le défis d'une effectivité » In konrad Stiftung, Naïrobie 2010, p. 20.

7 (2)F. TOENGAHO LOKUNDO, partis politique et décentralisation territoriale en R.D. Congo. Cas de la Province Orientale avant, SPA, Kisangani 2003, p. 73. 
- La décentralisation technique, celle qui porte sur les services ou les institutions outre que les entités géographiques (SNEL, REGIDESO, Université, Banque Centrale, ...)

- La décentralisation territoriale ou géographique qui s'applique aux entités territoriales ou subdivisions géographiques d'un Etat (Province, Ville, Commune, Secteur, ...)

Selon la doctrine administrative, la réalisation de la décentralisation territoriale est subordonnée au respect de trois conditions ${ }^{8}(3)$ :

$1^{\circ}$ Elle implique en premier lieu que soit déterminée une sphère des compétences spécifiques au bénéfice des collectivités locales : les affaires provinciales ou locales distinctes des affaires nationales.

$2^{\circ}$ En second lieu, elle suppose que ces affaires locales soient prises en charge par des autorités locales indépendantes du pouvoir central tant pour leur nomination que pour leur révocation.

$3^{\circ}$ En troisième lieu, la réalisation de décentralisation exige que cette gestion des affaires locales par les autorités locales soit autonome (libre administration).

\section{La Centralisation}

La décentralisation est le système d'organisation qui consiste à situer le pouvoir de décision au sommet de l'Etat, c'est-à-dire au niveau de l'autorité centrale dans la capitale, les autres collectivités ne pouvant agir que par délégation ${ }^{9}(1)$.

Elle est présentée au plan politique comme un moyen considéré et de maintenir l'autorité de l'Etat en évitant la dispersion des pouvoirs de réaliser et de sauvegarder l'unité nationale. Du point de vue administratif et pratique, elle est considérée comme un moyen d'assurer la régulation et l'impartialité dans l'allocation ou distribution des services et l'octroi des avantages aux administrés par l'aménagement de la gestion étatique au niveau natio$\mathrm{nal}^{10}(2)$.

En effet, la centralisation concentre l'ensemble des tâches administratives qui se superposent sur le territoire national entre les mains de l'Etat, il les dessine par une administration hiérarchique et unifiée. La particularité de ce système est donc que toutes les affaires publiques relèvent de l'Etat ou du pouvoir central administratif qui les gère à travers les différents services publics dépendant directement de lui.

Ainsi, la centralisation ne reconnaît aux collectivités locales aucune vie juridique, car celles-ci sont dépourvues de la personnalité juridique.

Elles ne sont en fait que des simples découpages administratifs et territoriaux, des circonscriptions administratives, c'est-à-dire de simple cadre destiné à permettre une implantation rationnelle des services de l'Etat sur l'ensemble de territoire ${ }^{11}(3)$.

8 (3) J. BANGUENARD, La décentralisation, peut paris 1980, pp 40-42.

9 (1)VUNDUAWE TE PEMAKO, Traité de Droit administratif, éd. Larcier Bruxelles, 2007, p. 405.

10 (2)NTAMBA LUABA LUMU, Droit constitutionnel général, E.U.A. Kinshasa 2005.

11 (3)VUNDUAWE TE PEMAKO, op. cit., p. 405. 
Le mécanisme de la centralisation s'est justifié par diverses raisons qui sont liées soit à la situation politique, soit à la situation économique et sociale. Dans le cadre de notre travail, nous sommes exonérés de ces détails.

Mais il convient de préciser que dans la centralisation, l'Etat est la seule personne publique pour l'ensemble du territoire national. De cette façon, il assume seul, sur son budget et par agent, la satisfaction de tous les besoins d'intérêt général ${ }^{12}(1)$. Deux modalités sont possibles dans la centralisation : il y a donc la centralisation centrée ou la conceptualisation et la centralisation alternée déconcentrée ou la déconcentration.

\section{Le régionalisme constitutionnel}

La décentralisation territoriale est en principe organisée et garantie par la loi jusque-là. On est en face de la décentralisation administrative simplement.

Le régionalisme constitutionnel va très loin dans le sens d'un desserrement ou relâchement des contraintes étatiques mais sans pour autant utiliser la forme fédérale de l'Etat. L'autonomie laissée à certaines collectivités locales ou à toutes, dépasse le niveau de la simple décentralisation et se trouve ou consignée dans la constitution avec une décentralisation des matières ${ }^{13}(1)$.

Le concept « régionalisme » est entré dans le vocabulaire juridique et politique dans la seconde moitié du $19^{\text {ème }}$ siècle. Mais ce n'est que vers le milieu du $20^{\text {ème }}$ siècle qu'il est d'usage élargi ${ }^{14}(2)$.

Dans le régionalisme constitutionnel la garantie de l'autonomie est constitutionnelle, les «provinces ou régions » sont affirmées comme des entités autonomes par la constitution. Celle-ci définit elle-même les matières qui relèveront de la compétence de ces entités, le législateur ne pourra réduire ou porter atteinte à l'autonomie reconnue par le constituant.

Ce modèle a été dégagé par la doctrine à partir du système italien, espagnol, portugais, belge (avant bien sûr la transformation de la Belgique en Etat fédéral en 1997).

Le régionalisme politique ou constitutionnel se caractérise par la reconnaissance d'une réelle autonomie politique au profit des entités régionales et notamment d'un pouvoir normatif autonome. Ainsi,, le régionalisme constitutionnel implique nécessairement le régionalisme politique en ce sens que les «provinces ou régions » bénéficiant constitutionnellement d'une autonomie politique, disposent d'un gouvernement (provincial) et d'une assemblée (provinciale) $)^{15}(3)$.

La régionalisation politique implique l'établissement de collectivités politiques, territoriales dans les régions, sur la base d'un principe de représentativité démocratique et d'auto

12 (1)Idem, p. 406.

13 (1)NTUMBA LUABA LUMU, op cit, p. 413.

14 (2)KAMUKUNY MUKINAY A, «Régionalisme, décentralisation et naissance effective des vingtcinq nouvelles provinces en R.D.C. défis et perspective des préventions des conflits », in CongoAfrique, $n^{\circ} 434$ Kinshasa, 2009, p. 21.

15 (3)VUNDUAWE TE PEMAKO, op. cit., p. 416. 
gouvernement. C'est pourquoi la régionalisation politique est elle-même un résultat de la décentralisation politique dans les Etats unitaires sur la base des régions ${ }^{16}(1)$.

En tout cas, ce qui caractérise le régionalisme est le fait que, en plus du niveau national qui conserve le pouvoir constitutif, unique et du niveau local (municipal), sont apparues des entités politiques, territoriales autonomes intermédiaires, produit de la décentralisation politique, dotées d'autonomie territoriale.

Ces unités territoriales intermédiaires se caractérisent par le fait d'avoir une autonomie politique avec le pouvoir législatif régional, dont les organes sont élus par vote populaire, ce qui matérialise la démocratie régionale est un instrument de décentralisation politique $^{17}(2)$.

C'est pourquoi le signe caractéristique contemporain des Etats unitaires est celui de leur régionalisation politique, engendrant une nouvelle forme d'Etat qui commence à se profiter : c'est celle de l'Etat régional ${ }^{18}(3)$.

\section{ATTENTES, ENJEUX ET PERSPECTIVES}

La décentralisation se mesure par le degré de liberté dont disposent les collectivités locales au sein d'un Etat unitaire. Elle constitue un point d'équilibre relativement instable et fragile résultant d'un compromis dynamique entre les forces centripètes qui poussent au renforcement de l'unité étatique et les forces centrifuges qui stimulent l'épanouissement de la diversité locale.

Expression précaire d'un rapport des forces évolutives, la décentralisation doit, pour exister, bénéficier conjointement de la vigueur des collectivités internes et de la complicité bienveillante du pouvoir central.

Selon la doctrine administrative, la réalisation de la décentralisation territoriale est subordonnée au respect des trois conditions ${ }^{19}(6)$.

1. Elle implique en premier lieu, que soit déterminée une sphère des compétences spécifiques au bénéfice des collectivités locales : les affaires provinciales ou locales distinctes des affaires nationales;

2. En second lieu, elle suppose que ces affaires locales soient prises en charge par des autorités locales indépendantes du pouvoir central tant pour leur nomination que pour leur révocation;

3. En troisième lieu, la réalisation de la décentralisation exige que cette gestion des affaires locales par les autorités locales soit autonome (libre administration).

Il convient de signaler qu'une décentralisation parfaite n'est pas sans danger, car les entités décentralisées, dotées de la personnalité juridique propre, de l'autonomie organique et de

16 (1)ALLAIN R \& BREWER CARAIS, Etudes de droit public comparé, éd. Bruxelles, 2001, p. 352.

17 (2) ALLAIN R \& BREWER CARAIS, op cit, p. 352.

18 (3)Ibidem, p. 356.

19 (6)J. BANGUENARD, la décentralisation, PUF, Paris, 1980, pp. 40-42. 
gestion, risqueraient de confondre leurs intérêts ou ceux de leurs populations locales avec l'intérêt général ou national et d'ignorer les lois de la République.

Pour éviter toute confusion de ce genre et sauvegarder l'intérêt général et la légalité au sein des entités territoriales décentralisées, le Gouvernement central prend toujours soin de conserver un droit de regard sur la gestion de ces entités. Ce pouvoir de contrôle ou de surveillance porte le nom de « tutelle administrative ». C'est ainsi que « dans un Etat unitaire, la décentralisation ne va pas sans tutelle, ni la tutelle sans décentralisation ». De même, il n'y a pas de tutelle sans texte, ni de tutelle au-delà des textes ${ }^{20}(7)$.

Ainsi définie, la nation de décentralisation se rapproche du fédéralisme, sans se confondre avec lui.

\section{DECENTRALISATION, FEDERALISME OU REGIONALISME}

La nature de la réforme de l'administration territoriale de 2008 consacrée par le bloc de trois lois précitées nécessite une clarification. En effet, l'article 2, alinéa 1 de la constitution du 18 février 2008 déclare à son alinéa $1^{\mathrm{er}}$ que : «La République Démocratique du Congo est composée de la Ville de Kinshasa et de 25 provinces dotées de la personnalité juridique », avant de préciser à l'alinéa 4 que « la répartition des compétences entre l'Etat et les provinces s'effectue conformément aux dispositions du titre III de la présente constitution ».

Par ailleurs, l'article 3 de la même loi-fondamentale dispose que : « Les provinces et les entités territoriales décentralisées de la République Démocratique du Congo sont dotées de la personnalité juridique et sont gérées par les organes locaux. Ces entités territoriales décentralisées sont : la ville, la commune, le secteur et la chefferie. Elles jouissent de la libre administration et de l'autonomie de gestion de leurs ressources économiques, humaines, financières et techniques $»$.

L'énumération des entités territoriales décentralisées soulève un problème sur le statut de l'entité « province » qui n'est pas reprise sur cette liste. La réponse à ce problème a été donnée par la loi $n^{\circ}$ 08/012 du 31 juillet 2008 qui, en son article 2, définit la province comme « une composante politique et administrative du territoire de la République ». Elle exerce, par ses institutions politiques, les compétences qui lui sont dévolues par la constitution.

20 (7)L. MASPERTIO \& P. LARAQUE, La tutelle administrative. Le contrôle des administrations départementales, locales et des établissements publics, cité par P. MAHON, la décentralisation administrative, Genève, p. 51. 
De ce qui précède, d'aucuns se posent la question de savoir sur quoi porte en réalité la reforme. S'agit-il :

- D'une réforme sur la décentralisation dans un Etat unitaire?

- D’une affirmation voilée du fédéralisme en consacrant l'autonomie politique des provinces? ou

- D’une simple régionalisation constitutionnelle?

Notre objectif est de faire connaitre au public les idées maîtresses que renferme le bloc des lois sur la décentralisation et, ainsi, contribuer à leur vulgarisation. Il s'agit également pour nous des faire savoir que sans une volonté politique affichée des décideurs au niveau central et sans mesures d'encadrement ou d'application adéquates, la reforme ainsi décrétée risque de demeurer un vœu pieux.

\section{LE REGIONALISME POLITIQUE}

Si la décentralisation ne peut être confondue avec le fédéralisme, elle n'est pas non plus synonyme de régionalisme politique ou constitutionnel.

Celui-ci constitue également un procédé d'aménagement du pouvoir d'Etat qui consiste à décentraliser politiquement et constitutionnellement les provinces qui, dans un Etat unitaire, deviennent des composantes politiques et administratives dotées de la personnalité juridique et de l'autonomie institutionnelle et financière ${ }^{21}(9)$.

Par rapport à la décentralisation, l'originalité du régionalisme politique réside dans le fait que la répartition des compétences entre le pouvoir central et les provinces ainsi que l'ordre institutionnel de celles-ci sont définis par la constitution et non par une loi organique qui peut, à tout moment, être modifiée par la seule volonté du législateur. Pour éviter les interventions intempestives du centre sur la périphérie, la loi fondamentale prend soin de distinguer clairement, un peu comme dans le fédéralisme :

- Les matières de la compétence exclusive du pouvoir central (matières liées à la souveraineté et celles d'importance nationale);

- Les matières de la compétence concurrente (nécessitant le concours de deux niveaux central et provincial pour une gestion efficace) et

- Cependant, à la différence des Etats fédérés, les provinces, dans un régionalisme politique ou constitutionnel ne jouissent pas de la liberté de se doter chacune de sa propre constitution et de son organisation interne. Et, à la différence d'un Etat unitaire décentralisé, le pouvoir central n'exerce pas la tutelle administrative sur les provinces dans le cadre du régionalisme politique.

21 (9)J. MEKHANTAR, Droit politique et constitutionnel, Paris, 1997, p. 51. Lire aussi X. PHILIPPE, «La répartition des compétences entre l'Etat central, l'Etat provincial et les municipalités ou administratives? » in Revue française de l'administration publique, $n^{\circ} 85$, janvier-Mars 1998, pp. $15-34$. 
En définitive, le régionalisme constitutionnel traduit clairement ce que d'aucuns appellent « un Etat unitaire fortement décentralisé ». C'est donc une formule de gestion intermédiaire entre un Etat unitaire décentralisé et un Etat fédéral.

Mais, les trois systèmes de gouvernement (décentralisation, fédéralisme et régionalisme politique) reposent tous sur le principe de la libre administration.

La différence entre décentralisation, fédéralisme et régionalisme politique étant clairement établie. Revenons maintenant à la question de savoir sur quoi porte la reforme de l'administration territoriale de 2008 en République Démocratique du Congo et quelles sont ses principales innovations.

\section{L'INSTAURATION SIMULTANEE DE TROIS MODES DE GESTION DES ENTITES TERRITORIALES}

La réforme de l'administration territoriale consacrée par le bloc de lois précitées institue trois modes différents de gestion des entités territoriales, à savoir :

- Le régionalisme politique qui repose sur le principe de la libre administration des provinces;

- La décentralisation territoriale qui s'applique aux villes, communes, secteurs et chefferies et

- La décentralisation qui, elle, s'applique aux territoires, quartiers, groupements et villages.

\section{LA REGIONALISATION DES PROVINCES}

Aux termes de la loi $\mathrm{n}^{\circ}$ 08/012 du 31 juillet 2008 portant principes fondamentaux relatifs à la libre administration des provinces, la province est une composante politique et administrative du territoire de République (article 2). Elle est dotée de la personnalité juridique et jouit de l'autonomie de gestion de ses ressources humaines, économiques, financières et techniques (Assemblée provinciale et Gouvernement provincial) les compétences qui lui sont dévolues par la constitution. C'est ce qui lui confère une autonomie politique.

Pour éviter que chaque province bénéficiant d'une autonomie politique et financière ne puisse mener sa politique en vase clos, la reforme a institué la conférence des Gouverneurs de province. Celle-ci est un cadre de concertation régulière entre les provinces et le pouvoir exécutif national. Sa mission est d'émettre les avis et de formuler des suggestions concrètes sur la politique à mener et la législation à élaborer. Elle participe à la consolidation de l'unité, de la paix et de la solidarité nationale et assure une bonne harmonie entre le pouvoir exécutif national et les provinces, d'une part, et celles-ci entre elles, d'autre part. Mais, sa composition et son fonctionnement suscitent quelques inquiétudes. 


\section{LES AUTRES TRAITS FONDAMENTAUX DE LA DECENTRALISATION TERRITORIALE}

La reforme consacre aussi :

1. Une répartition judicieuse des compétences entre les différentes entités territoriales décentralisées afin de leur assurer un développement harmonieux.

2. La libre administration d'une entité décentralisée dans la mesure où elle décide librement dans la sphère des compétences qui lui sont conférées sans immixtions de l'autorité provinciale, sauf dans les cas limitativement énumérés par la loi.

3. Le principe de la représentation en même temps de l'Etat et de la province par les autorités exécutives locales assure également la coordination et le suivi des services de l'Etat et de la province dans leurs entités respectives. L'exercice des compétences déconcentrées de l'Etat se fait sous l'autorité du Gouverneur qui peut déléguer ses pouvoirs à l'Administrateur de territoire.

4. L'autonomie financière qui permet à une entité territoriale décentralisée de disposer d'un budget propre, distinct de ceux du pouvoir central et de la province. Ce budget est toutefois intégré en dépenses et en recettes au budget de la province qui est présenté en même temps que le budget du pouvoir central pour former le budget de l'Etat arrêté chaque année par une loi.

5. Le droit des entités territoriales décentralisées à $40 \%$ des recettes à caractère national rétrocédées à la province ainsi que la possibilité de bénéficier des ressources de la caisse de péréquation.

Il convient également d'indiquer qu'une entité territoriale décentralisée dispose de ressources exceptionnelles. Il lui est toutefois interdit de recourir aux emprunts extérieurs.

L'autorité exécutive d'une entité territoriale décentralisée est placée sous la tutelle du Gouverneur de province. Celui-ci exerce un contrôle à priori ou à posteriori sur les actes.

Pour leur garantir le libre exercice des compétences que leur reconnaissent la constitution et les lois, les membres des organes délibérants d'une entité territoriale décentralisée, jouissent des immunités contre les poursuites judiciaires dans les limites des dispositions de l'article 107 de la constitution.

En outre, la loi institue un privilège de juridiction au bénéfice de toutes les autorités d'une entité territoriale décentralisée. En matière pénale, elles sont, selon le cas, judiciables de la cour d'appel ou d'un tribunal de Grande Instance en premier ressort.

Les autorités exécutives locales représentent le pouvoir central dans leurs juridictions respectives. Elles exécutent les lois, édits et règlements nationaux ou notamment des forces de la Police Nationale mises à leur disposition. 
Tels sont les traits essentiels qui constituent en même temps les points forts de la réforme qui consacre la décentralisation de certaines entités territoriales de la République Démocratique du Congo $22(14)$.

Comme on peut le constater, le sort des entités territoriales déconcentrées (territoires, quartiers, groupements et villages) ainsi que leurs rapports avec les E.T.D., demeurent encore inconnus en attendant la proposition de la loi encore en étude au niveau de deux chambres du parlement : Voilà ce qui traduit l'idée d'une reforme à deux vitesses.

\section{LES FAIBLESSES DE LA REFORME}

Si la présente reforme peut être considérée comme une avancée significative de la marche de l'administration territoriale congolaise vers une véritable territoriale de développement, il y a lieu de reconnaître aussi qu'elle ne manque pas de faiblesse et que sa mise en œuvre poserait quelques problèmes. Parmi ceux-ci, nous pouvons relever à titre indicatif :

- à la libre administration des provinces;

- à la conférence des gouverneurs de province;

- aux entités territoriales décentralisées (villes, communes, secteurs et chefferies);

- aux entités territoriales déconcentrées.

A cet effet, le législateur congolais aurait pu s'inspirer utilement des lois antérieures, notamment la loi $n^{\circ} 082 / 006$ du 25 février 1982 portant organisation territoriale, politique et administrative de la République du Zaïre qui, au-delà de son contenu, avait le mérite d'unifier la législation sur l'organisation de la territoriale congolaise dans un texte unique.

\section{LE DEDOUBLEMENT FONCTIONNEL DES GOUVERNEURS DE PROVINCE}

Le dédoublement statut du Gouverneur de province qui est à la fois le chef de l'exécutif et l'autorité provinciale élue, responsable devant l'assemblée provinciale, d'une part, et le représentant du Gouvernement central chargé de coordonner les services publics déconcentrés en province et répondant de ses actes devant le gouvernement central par l'intermédiaire de l'assemblée provinciale, d'autre part, est un véritable goulot d'étranglement à la libre administration de la province.

Comment l'Assemblée provinciale sera-t-elle en mesure de contrôler efficacement et de sanctionner en toute indépendance le gouverneur des fautes qu'il aura commises en sa qualité de représentant du gouvernement central surtout quand on sait que le choix de ce gouverneur ainsi que celui des membres du bureau de l'assemblée provinciale ont été dictés par le pouvoir central?

22 (14)Il convient de signaler que ces éléments sont clairement exprimés dans l'exposé des motifs de la loi organique $n^{\circ}$ 08/016 du 07 octobre 2008 portant composition, organisation et fonctionnement des entités territoriales décentralisées et leurs rapports avec l'Etat et les provinces. 
Ce dédoublement fonctionnel du gouverneur risque d'affaiblir l'assemblée provinciale qui aura beaucoup de gêne et d'hésitation dans l'exercice de sa mission de contrôle de l'exécutif provincial.

\section{L'INSTAURATION D'UNE CARRIERE POUR LES CADRES DE LA TERRITORIALE DEBOUT}

La suppression sur la carte administrative de la République Démocratique du Congo de l'échelon « District » et l'imposition de l'élection comme seul mode d'accession aux fonctions de gouverneur de province consacrent du coup la politisation de la territoriale et l'instauration d'une carrière plane pour les administrateurs de territoire et leurs assistants. Ces derniers se voient ainsi privés de toute possibilité de promotion dans la territoriale débout, ce qui ne constitue pas moins un facteur de démotivation pour cette catégorie d'agents publics dont on a pourtant grandement besoin pour une régulation de l'administration publique, en général, et de la territoriale, en particulier, a déjà démontré ses limites par le passé.

\section{L'ERECTION DU CHEF-LIEU DU TERRITOIRE EN COMMUN}

En érigeant l'actuelle « cité » qui sert de Chef-lieu du territoire en commun, une entité territoriale décentralisée, la reforme décrétée ouvre les perspectives d'un conflit inévitable entre l'administrateur de territoire (autorité déconcentrée) et le bourgmestre de la nouvelle commune chef-lieu du territoire (autorité décentralisée).

Pour éviter ce conflit, certaines mesures règlementaires clarifient les rapports entre ces deux autorités ayant des grades statutaires équivalents s'imposent.

\section{CONCLUSION}

Aux termes de la loi $n^{\circ}$ 08/012 du 31 juillet 2008 portant principes fondamentaux relatifs à la libre administration des provinces, la province est une composante politique et administrative du territoire de la République (article 2). Elle est dotée de la personnalité juridique et jouit de l'autonomie de gestion de ses ressources humaines, économiques, financières et techniques. Elle exerce par ses institutions politiques (Assemblée provinciale et Gouvernement provincial) les compétences qui lui sont dévolues par la constitution. C'est ce qui lui confère une autonomie politique.

Pour éviter que chaque province bénéficiant d'une autonomie politique et financière ne puisse mener sa politique en vase clos, la reforme a institué la conférence des gouverneurs de province. Celle-ci est un cadre de concertation régulière entre les provinces et le pouvoir exécutif national. Sa mission est d'émettre les avis et de formuler des suggestions concrètes sur la politique à mener et la législation à élaborer. Elle participe à la consolidation de l'unité, de la paix et de la solidarité nationale et assure une bonne harmonie entre d'une part, et 
celles-ci entre elles, d'autre part. Mais, sa composition et son fonctionnement suscitent quelques inquiétudes.

Par ailleurs, le système actuel et l'application de la décentralisation dans notre pays révèle que le système centralisé met toujours son ombre dans la politique et la gestion du pays.

De ce qui précède, il convient de noter que l'application de la constitution à la lettre pose problème dans la mesure où, alors que la constitution parle de la retenue à la source, d'autres lois organiques parlent en même temps de la rétrocession, ce qui prouve à suffisance que le droit des ETD est bafoué et réduire au centralisme à outrance.

Cependant, le régionalisme politique qui repose sur le principe de la libre administration des provinces révèle que le découpage territorial apparaît comme un mécanisme de régulation de tension générée par le conflit de leadership surtout au niveau national et provincial de part leurs institutions, alors que le découpage territorial est encore une utopie.

Il faut relever, cependant que, le système centralisé persiste encore dans l'application de la constitution étant donné que les entités territoriales décentralisées ne bénéficient plus directement de la rétrocession de la part du gouvernement provincial, de même celui-ci du gouvernement central. Ce qui nous permet de souligner que malgré toutes les tentatives de l'application de la décentralisation, le système centralisé bat toujours son épanouissement dans la gestion de la République.

A l'issue de nos enquêtes sur le terrain, il ressort qu'après l'amorce du processus de décentralisation territoriale, le constituant du 18 février 2006 a viré vers le régionalisme constitutionnel, sans le dire expressément, mais le définit à travers des dispositions constitutionnelles nettes et sans équivoque, du moins pour le politologue perspicace et non proclamer l'unicité et l'indivisibilité de l'Etat Congolais. Elle crée ensuite au sein de l'Etat, de subdivisions territoriales inféodées, pourvues d'une personnalité morale et d'une autonomie différente de degré selon qu'elle est directement garantie par la constitution pour les unes et assurée par le législateur pour les autres.

Le régionalisme politique congolais, devrait être effectif, être accompagné d'un transfert total des compétences et d'un véritable relâchement par le pouvoir central de certaines de ses prérogatives.

Le pouvoir central, jusqu'à ce jour, n'a pas respecté le principe de la retenue à la source des $40 \%$ de l'ensemble des recettes à caractère national, dévolues aux provinces à l'article 175 de la constitution. Bien plus encore, la caisse nationale de péréquation dont la mission consiste à financer des projets et programmes d'investissement public, en vue d'assurer la solidarité nationale et corriger le déséquilibre de développement entre les provinces et entre les entités territoriales décentralisées n'est pas encore créée.

\section{BIBLIOGRAPHIE}

1. ALLAIN R \& BREWER CARAIS, Etudes de droit public comparé, éd. Bruxelles, 2001. 
2. BAGAHUA MUHEME, Economie régionale par la gestion décentralisée des projets. Academia Bruylant, Louvain-la-Neuve 2005.

3. F. TOENGAHO LOKUNDO, partis politique et décentralisation territoriale en République Démocratique du Congo. Cas de la Province Orientale avant, SPA, Kisangani 2003.

4. Félix VUNDUAWE té PEMAKO, la dynamique de la décentralisation territoriale en République Démocratique du Congo, Afrique n 433, Mars 2009, Lubumbashi, pp. 165 à 185.

5. J. BANGUENARD, La décentralisation, Paris, PUF, 1980.

6. J. MEKHANTAR, Droit politique et constitutionnel, Paris, 1997, p. 51. Lire aussi X. PHILIPPE, « La répartition des compétences entre l'Etat central, l'Etat provincial et les municipalités : Structures politiques ou administrative? » In revue française de l'administration publique, $\mathrm{n}^{\circ} 85$, JanvierMars 1998, pp. 15-35.

7. J. MEKHANTAR, Droit politique et constitutionnel, Paris, 1997.

8. KAMUKUNY MUKINAY A, « Régionalisme, décentralisation et naissance effective des vingt-cinq nouvelles provinces en R.D.C. défis et perspective des préventions des conflits ", in CongoAfrique, $n^{\circ} 434$ Kinshasa, 2009, p. 21 et suiv.

9. KAPYA KABESA « la décentralisation au Ruanda, au Burundi et en R.D.C., le défis d'une effectivité » In konrad sciftung, Naïrobie 2010, p. 20 et suiv..

10. L. MASPERTIO \& P. LARAQUE, La tutelle administrative. Le contrôle des administrations départementales, locales et des établissements publics, cité par P. MAHON, la décentralisation administrative, Genève.

11. loi organique $n^{\circ} 08 / 016$ du 07 octobre 2008 portant composition, organisation et fonctionnement des entités territoriales décentralisées et leurs rapports avec l'Etat et les provinces.

12. NTUMBA LUABA LUMU, Droit constitutionnel général, E.U.A. Kinshasa 2005.

13. Simon-Pierre METENA M'NTEBA, Des entités territoriales décentralisées (E.T.D.) qu'est-ce à dire et pourquoi faire? In Congo-Afrique $\mathrm{n}^{\circ}$ 433, Mars 2009, L'shi, pp. 187- 202.

14. Simon-Pierre METENA, Des « Entités territoriales décentralisées (E.T.D.) ». Qu'est-ce à dire et pourquoi faire? In Congo Afrique n 433 mars 2009, Lubumbashi, pp. 187 à 202.

15. VUNDUAWE TE PEMAKO, Traité de Droit administratif, éd. Larcier Bruxelles, 2007.

16. X. PHILIPPE, « La répartition des compétences entre l'Etat central, l'Etat provincial et les municipalités ou administratives? » in Revue française de l'administration publique, $n^{\circ} 85$, janvier-Mars 1998, pp. 15-34. 


\section{Impressum}

\section{KAS African Law Study Library - Librairie Africaine d'Etudes Juridiques}

Herausgeber: Konrad-Adenauer-Stiftung e.V., Klingelhöferstraße 23, 10785 Berlin

Schriftleitung: RA Prof. Dr. Hartmut Hamann, Falkertstraße 82, D-70193 Stuttgart Telefon: +49 (o) 711 120950-30 | Fax : +49 (o) 711 120950-50

Mail:hamann@hamann-legal.de

Erscheinungsweise: 4 Ausgaben pro Jahr

Druck und Verlag: Nomos Verlagsgesellschaft mbH \& Co. KG - Waldseestr. 3-5 D-76530 Baden-Baden. Telefon +49-7221-2104-0 / Fax 49-7221-2104-27 E-Mail:nomos@nomos.de

Anzeigen: sales friendly Verlagsdienstleistungen - Inh. Frau Bettina Roos - Pfaffenweg 15 - D-53227 Bonn Telefon +49-228-97898-o / Fax +49-228-97898-20 E-Mail:roos@sales-friendly.de

Urheber- und Verlagsrechte: Die Zeitschrift und alle in ihr enthaltenen einzelnen Beiträge sind urheberrechtlich geschützt. Jede Verwertung außerhalb der engen Grenzen des Urheberrechtsgesetzes ist ohne Zustimmung des Verlages unzulässig. Das gilt insbesondere für Vervielfältigungen, Übersetzungen, Mikroverfilmungen und die Einspeicherung und Verarbeitung in elektronischen Systemen. Der Nomos Verlag beachtet die Regeln des Börsenvereins des Deutschen Buchhandels e.V. zur Verwendung von Buchrezensionen.

ISSN $2363-6262$

\section{Nomos}

